\title{
Surface Tracker: A prototypic model based on IoT and Cloud for Mars Expedition
}

\author{
Jithu Biju ${ }^{1}$, Haritha Sajikumar' ${ }^{2}$, Sujitha $\mathrm{M}^{3}$ \\ ${ }^{1}$ Mangalam College of Engineering, Department of Computer Science, Kottayam, India, jithubiju007@gmail.com \\ ${ }^{2}$ Mangalam College of Engineering, Department of Computer Science, Kottayam, India, harithasajikumar597@ gmail.com \\ ${ }^{3}$ Mangalam College of Engineering, Department of Computer Science, Kottayam, India, m.sujitha@mangalam.in
}

\begin{abstract}
Nowadays, Mars expeditions are gaining more significance as the world scientists are searching if the red planet can handle life as the blue planet doing. This paper presents a prototypic model that can be used in Mars expeditions. The prototypic model surface tracker is a hardware device equipped with sensors for sensing temperature, humidity, and moisture content of the surface to which it is deployed. For temperature and humidity, sensing the DHT22 sensor is used and FC-28 for moisture sensing. The sensed values are uploaded to IoT platform ThingSpeak and to the Blynk android app, the values are accessed on the screens of mobile or laptop. Based on the results, the analysis is done and if the analysis result is positive, the device will deploy the seeds which it carried, to that surface. The model is a prototype of the idea that can be implemented effectively in Mars expedition by making slight changes in prototype based on the communication technologies used in mars surface as the technologies supporting communication in earth and mars are entirelydifferent.
\end{abstract}

Key words: Cloud, Humidity, Internet of Things, Mars, Sensors, Temperature.

\section{INTRODUCTION}

In this developing era, countries have been focusing on expeditions to celestial bodies in the quest to investigate the possibilities of life outside the Earth. The query of humans towards gaining and finding out new details about celestial bodies is increasing day by day. Scientists all over the world were enthusiastic in solar explorations from early centuries too: Galileo Galilei made use of the telescope for observing night sky during the early decades of the 1600s. Celestial bodies have been always enthralling for humankind and men started to uncover the fascinating world of the heliosphere. As the technologies and opportunities to scientists kept on increasing day by day, discoveries have also increased which provided a long range of knowledge and scopes in explorations[1].

Prototypic model Surface Tracker uses sensors mainly. Sensors are the key components in any of IoT applications as they are able to collect real-time data and are widely used in many real-time applications, thus created numerous advantages in many fields. There are different types of sensors like LDR-light sensor, DHT11-temperature sensor, HCSR501-motion sensor, HCSR04-distance sensor, Galvanometercurrent sensor, MAX44005-colour and infrared proximity sensor, ultrasonic sensors etc. The selection of sensors entirely depends on the needs of the user. Any IoT application needs devices to react to data, some nodes for data collection and some hardware communication modules, gateways to connect over wireless and fixed networks to the internet. IoT, is the Internet of Things, where a large number of devices can be interconnected and can communicate or transfer data without human-tohuman or human- to-computer interaction. In the implementation of IoT applications, the basic foundation is smart sensors embedded with microcontrollers. In an IoT ecosystem, the very important two things are the internet and the physical devices like actuators and sensors where the bottom layer contains network to collect data and sensor connectivity. The sensed data from sensors are sent to cloud, as the sensors contains processors that are sophisticated to configure the data to be sent to the cloud. Cloud computing, the first services are barely a decade old, but now all are embracing this technology for some reasons. Cloud computing is evolving as future generations computer paradigm.. Another application of Cloud Computing lies in Intelligent Transportation System which is very efficient in solving transport related issues in smart cities [4]. Cloud computing provides services like Platform as a Service (PaaS), Infrastructure as a Service (IaaS), Software as a Service (SaaS) and also provides massive storage spaces.

\section{HARDWAREIMPLEMENTATION}

The hardware side of the proposed prototype consists of: 


\section{Microcontroller-NodeMCU ESP8266}

The microcontroller NodeMCU ESP8266 comes with an EEPROM CP2102 where it is an Open Source Wi-Fi Development Board. It is used for transmitting and receiving data and for controlling different components using(IEEE 802.11) Wi-Fi.

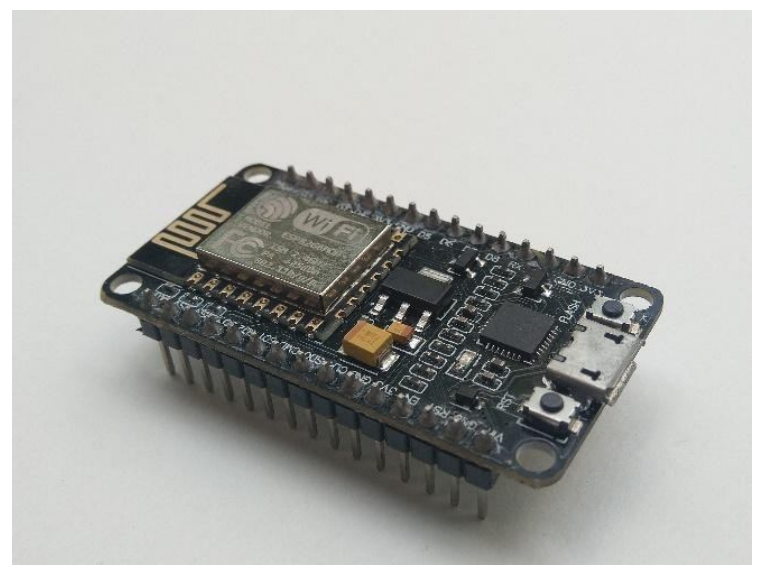

Figure 1: NodeMCU ESP8266.

\section{Arduino UNO}

ArduinoUNO is another Microcontroller which is used in this prototypic model where the main part of obstacle avoidance is done through this microcontroller and UltrasonicSensor.

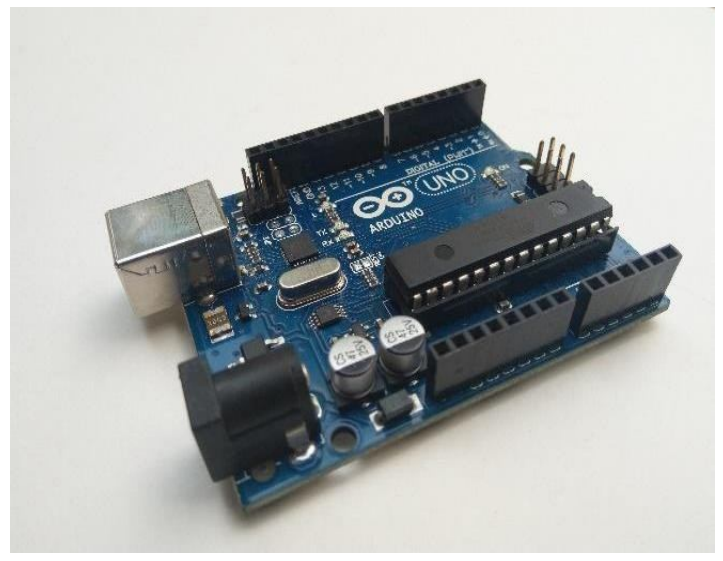

Figure 2: Arduino UNO.

\section{Temperature and humidity sensor}

DHT22 sensor can be used for sensing the temperature and humidity of the surface as these two factors play key roles in the germination of a seed.DHT22 sensor will be a better option for this prototype than DHT11F igure 3 as the former can decode the atmospheric temperature and humidity more accurately and rapidly. The DHT11 sensor can play vital role in the mode of crop irrigation and along with actuators will make it possible to lower the temperature and increase or decrease relative humidity as well as temperature depending on type of crop [1]. By using data output pin DOUT, which is connected to the SDA of AM2302, the sensed values are collected and uploaded to IoT platform ThingSpeak using AT commands and an android application to view the data.

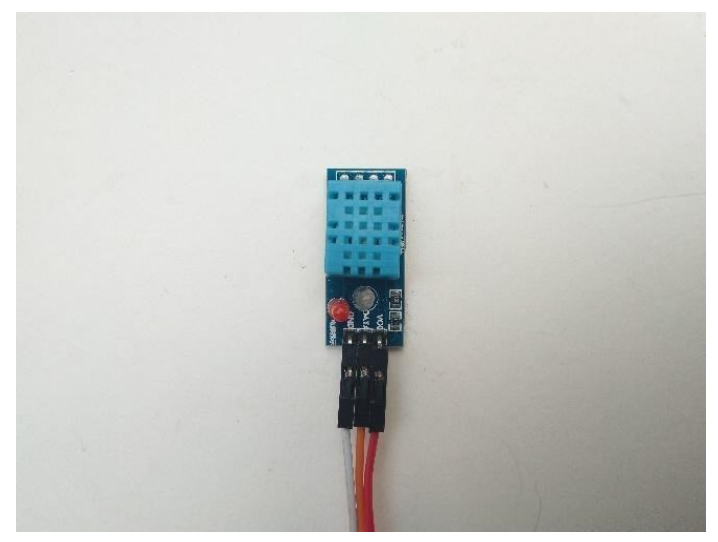

Figure 3:DHT11.

\section{Soil moisture sensor}

Moisture, being the presence of water content in the soil, is another essential factor for growth to take place. For sensing moisture content, FC-28 Figure 4 is used, which senses the moisture and theaccurate percentage of water content will bedisplayed on the app screen.

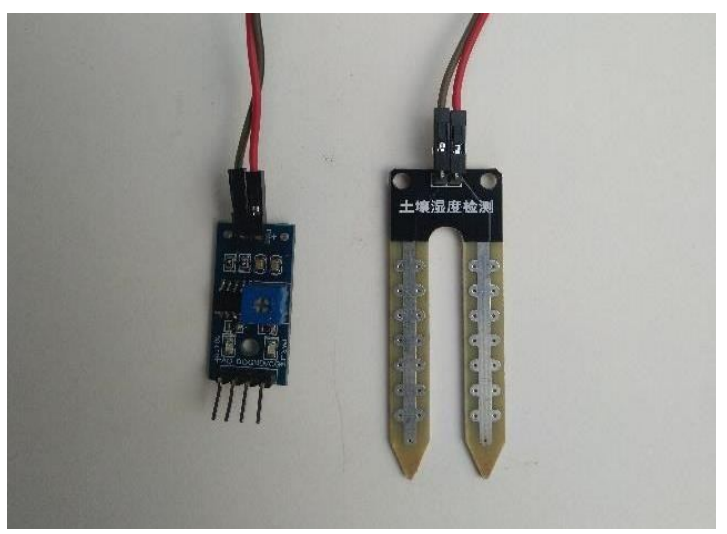

Figure 4: Soil moisture sensor.

FC-28 comes with a mini two-probe, which can easily penetrate through the soil, and the SG90 S Metallic servo makes the sensor penetrate to soil from enginechassis. When the electrodes are kept in soil, the moisture level changes can be measured in a relative manner[2]. 


\section{Ultrasonic sensor}

Ultrasonic sensors emit ultrasonic waves where it can detect and calculate the exact distance from an obstacle. The principle behind the calculation is that the two heads of the sensors transmit and receive the reflected waves from the obstacle and the time between the transmission and reception is calculated to find out the distance. By calculating the distance, the sensor can identify an obstacle in front of the surface tracker and take appropriate steps. The sensor used is an HC-SR04 ultrasonic Sensor Figure5.

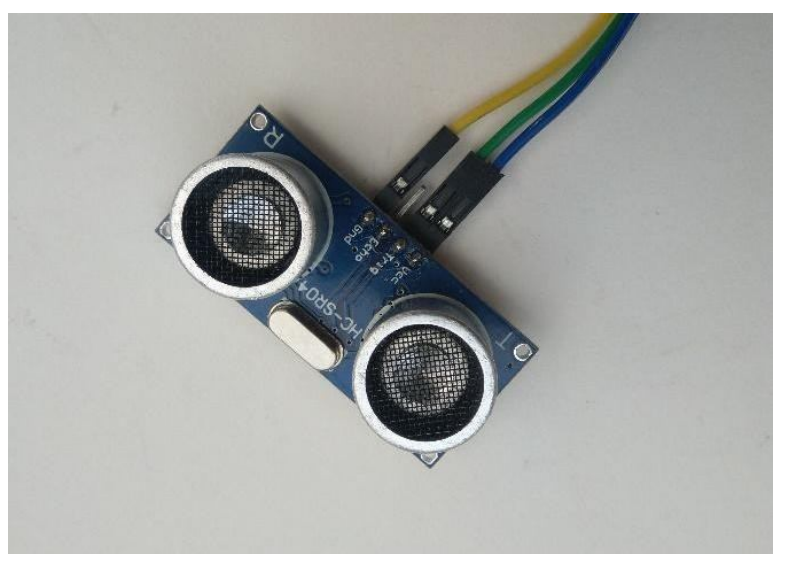

Figure 5:HC-SR04Ultrasonic Sensor

\section{WD Engine Chassis}

For the smooth movement in the surface of Mars and for collecting data using different sensors, 4WD engine chassis will help at its best where it comes with a four by four drive, which refers to a dual axis that can move on any terrain. The four-wheel drive is that the four wheels can provide torque at the same time thus making journeys smooth irrespective of the terrain.

\section{SYSTEMIMPLEMENTATION}

As a prototypic model, the data transferring between the components and cloud/IoT platform is done using Wi-Fi IEEE 802.11. The NodeMCU 8266 modules help to communicate with components where it consists of many digital pins and 1 analog pin.

- The digital pin D2 i.e. GPIO04 gets the data from the DHT22 sensor and the data is transmitted using $\mathrm{RxD}$ and TxD pins ofNodeMCU.

- The analog pin on the NodeMCU 8266 is A0, which gets the data from the moisture sensor, convert the analog data to digital data bythe module and transmit the values to the Blynk Application.

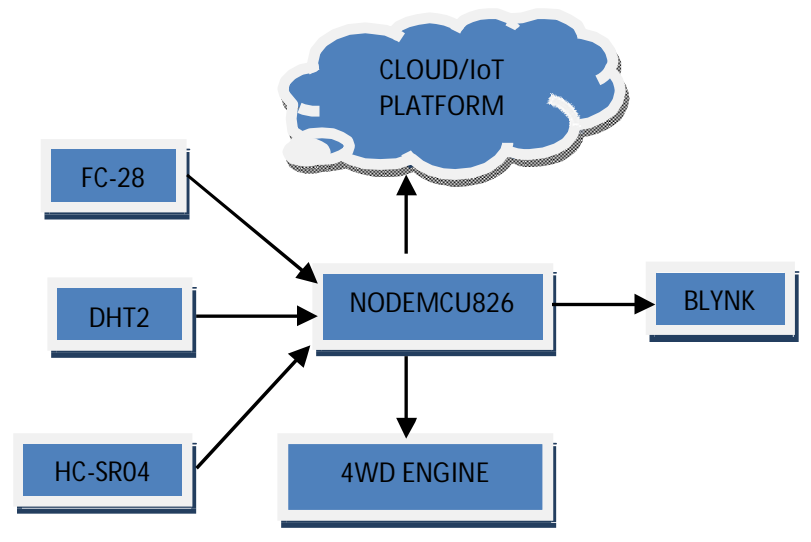

Figure 6: Block diagram of the system.

- HC-SR04 Ultrasonic Sensor is used to avoid the collision via obstacle avoidance. The trigger pin is connected to the Digital pinGPIO2 (D4) of NodeMCU and the Echo Pin is connected to the GPIO0(D3).

- The GND and VCC pins of DHT11, Soil Moisture sensor, and Ultrasonic sensor are connected to the NodeMCU module.

Figure 6 shows the block diagram of the hardware. As the device is on, the sensors start sensing atmospheric temperature and humidity, the values are uploaded using AT commands-AT+CIPSEND to the IoT platform ThingSpeak, where it shows the temperature and humidity values at real time in a graphical representation[3].

\section{RESULTS ANDDISCUSSIONS}

When the Surface Tracker is introduced into a surface, it can move freely irrespective of the terrain. Ultrasonic sensors are most suitable for obstacle detection and are widely used because of high ranging capacity and low cost. The sensor is attached at the front part of the device and whenever the device is going on the desired path, the ultrasonic sensor transmits ultrasonic waves continuously from its sensor head. Whenever an obstacle comes ahead of Surface Tracker the ultrasonic waves are reflected back from the obstacle and that information ispassed 
to the microcontroller. The microcontroller, in turn, controls the motors back, front, left, right and to control the speed of each motor Pulse Width Modulation (PWM) is used. The distance to the obstacle is calculated based on the reflected waves from the obstacle and distance is calculated using the formula (1)

\section{$\mathbf{L}=\mathbf{1 / 2} \times \mathbf{T} \times \mathbf{C}(1)$}

where $L$ is the distance to be calculated, $T$ is the time and $C$ is the speed.ThingSpeak also shows a comparison of temperature for the last 3 days by making use of the saved data using Blynk.

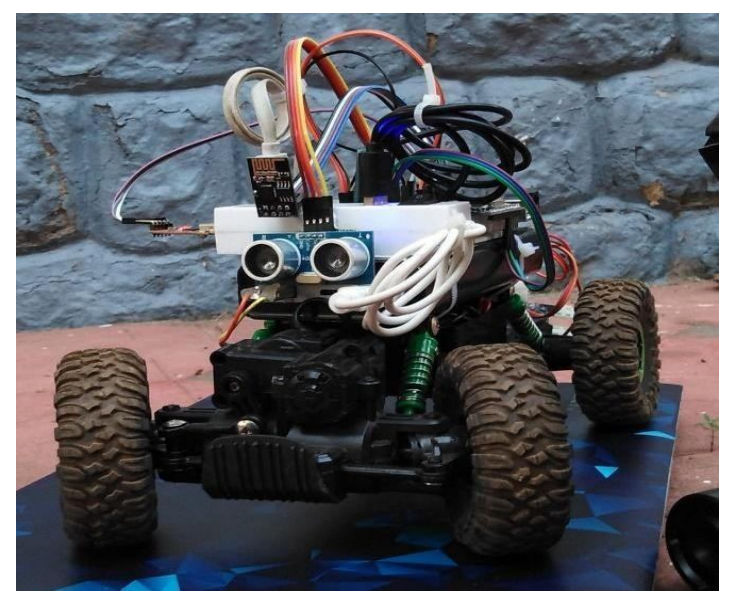

Figure 7: Proposed system.

There is a Correlation graph also present which provides the relationship between temperature and humidity. There are two types of correlations: variables that are a positively correlated move in the same direction-positive correlation and variables that are a negatively correlated move in opposite directions. Sensed values from sensors are also taken to android application Blynk. The function of message transfer part is reliable delivery between end users and network [7]. As the proposed system Figure 7 is a prototype only, currently the communications take place through Wi-Fi technology (IEEE 802.11). When coming to the implementation level, Wi-Fi will be replaced with satellite communication techniques and antennas for communication similar to how satellites sent images to earth [6].

The data which is collected by the sensors is uploaded to the ThingSpeak where it shows the graphical representation of both humidity and temperature with a three-day comparison graph and correlation graph are shown in Figure 8.
Also the same data is shown in the android application Blynk, where it shows the real-time percentage of moisture content and the exact value of humidity and temperature also with the control to sow seeds which are stored on the Surface tracker. Figure 9 shows the results from Blynk application.
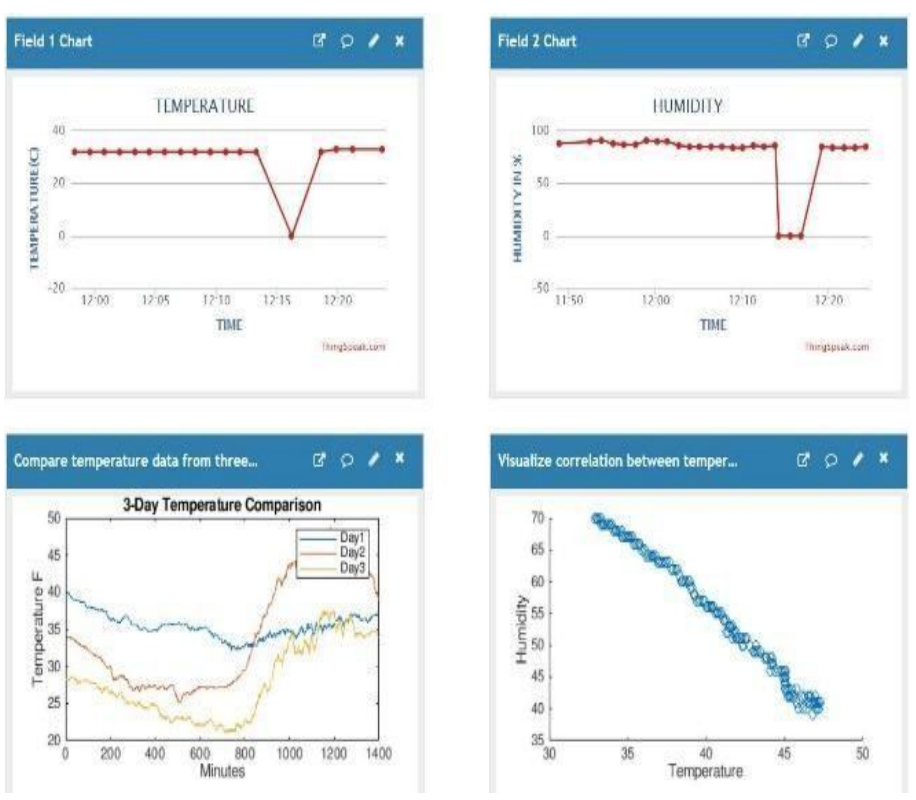

Figure 8: Graphical representation on the ThingSpeak.

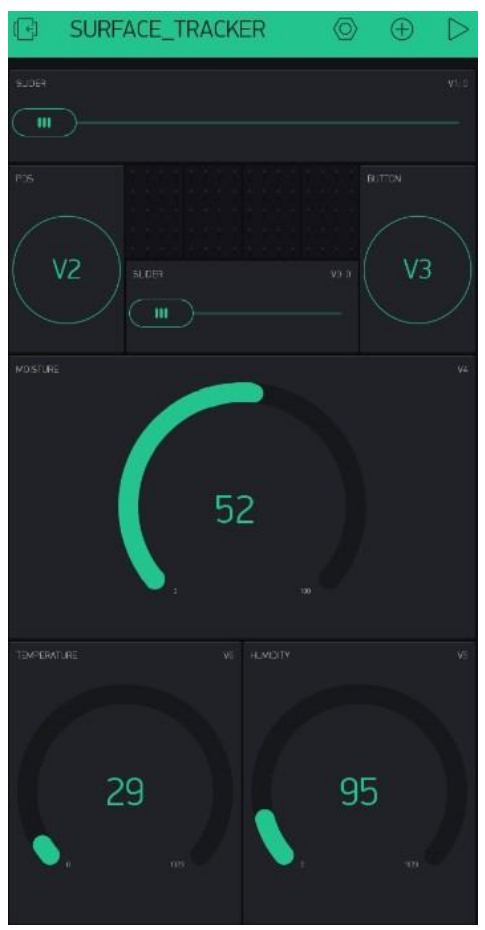

Figure 9: Result from the Blynk application. 


\section{FUTUREWORKS}

As the Surface Tracker is a prototype to Mars Expedition, it can be used in the agriculture field as an effective tool that would create a tremendous leap in traditional agriculture techniques existing in soil testing. Soil parameters such as moisture content, temperature,humidity and $\mathrm{pH}$ play a vital role in agriculture production. Real-time monitoring of soil parameters can be accomplished using appropriatesensors connected to the Internet using Wi-Fi connectivity [5]. Surface Tracker can sense the atmospheric conditions of the surface to which it is deployed. This methodology can be implemented in agricultural fields for soil testing and introducing a $\mathrm{pH}$ sensor to the existing model will provide the scope of testing soil $\mathrm{pH}$ too that will reduce human effortconsiderably.

\section{CONCLUSION}

Science and technology are growing day by day and by effective use of new technologies in all fields can produce huge leaps in the life of man. The prototype presented here uses technologies IoT, sensors and gives a model that can be used in the Mars expedition by applying slight changes. The model makes use of new generation technologies and makes effective results. In the future, this model can be used to produce significant changes in traditional agricultural methods of soil testing etc, thus reducing human effort and time considerably.

\section{REFERENCES}

[1] Carlos Andrés González-Amarillo, Juan Carlos CorralesMuñoz, Miguel Ángel Mendoza-Moreno,Angela maría González Amarillo, Ahmed Faeq Hussein, N. Arunkumar,Gustavo Ramirez-GonzálezAn, "IoT-based traceability system for greenhouse seedling crops"IEEE Access, on New Trends in Brain Signal Processing and Analysis (Volume: 6), 22 October 2018 DOI: 10.1109/ACCESS.2018.2877293.

[2] A. Kumar, K. Kamal, M. O. Arshad, S. Mathavan, T. Vadamala, "Smart irrigation using low-cost moisture sensors and XBee-based communication",IEEE Explore: 04 December 2014 DOI: 10.1109/GHTC.2014.6970301,

[3] Anis Koubaa, Basit Qureshi, "DroneTrack: Cloud-Based Real-Time Object Tracking Using Unmanned Aerial Vehicles Over the Internet"IEEE Access (Volume: 6)13810 13824,05March2018DOI:10.1109/ACCESS.2018.2811762.

[4] Aditi Zear, Pradeep Kumar Singh and Yashwant Singh, Indian Journal of Science and Technology, Vol 9(32), “IntelligentTransportSystem:AProgressiveReview”DO
I:10.17485/ijst/2016/v9i32/100713, August2016.

[5] P. Divya Vani and K. Raghavendra Rao, Indian Journal of Science and Technology, Vol 9(31), August 2016, "Measurement and Monitoring of Soil Moisture using Cloud IoT and Android System"DOI:10.17485/ijst/2016/v9i31/95340.

[6]Anil k Maini, Varsha Agrawal. "Satellite Communications" pp 168-169.

[7]Theodore S.Rappaport. "Wireless Communications principles and practice", Second edition, pp 518-519. 